supported by Deutsche Forschungsgemeinschaft (DFG), Sonderforschungsbereich (SFB) 914

Disclosure Information B. Uhl: None. L. Mittmann: None. J. Dominik: None. R. Hennel: None. B. Smiljanov: None. F. Haring: None. J. Schaubächer: None. C. Braun: None. L. Padovan: None. R. Pick: None. M. Canis: None. C. Schulz: None. M. Mack: None. E. Gutjahr: None. P. Sinn: None. J. Heil: None. K. Steiger: None. S.M. Kanse: None. W. Weichert: None. M. Sperandio: None. K. Lauber: None. F. Krombach: None. C. Reichel: None.

\section{P02.10 FOCUSCOPE: A SINGLE CELL, MULTI-OMICS SOLUTION TO SIMULTANEOUSLY ANALYZE TUMOR VARIANTS AND MICROENVIRONMENT}

Y Arjmand Abbassi*, N Fang, W Zhu, Y Zhou, Y Chen, U Deutsch. Singleron Biotechnologies GmH, Köln, Germany

10.1136/jitc-2021-ITOC8.22

Recent advances of high-throughput single cell sequencing technologies have greatly improved our understanding of the complex biological systems. Heterogeneous samples such as tumor tissues commonly harbor cancer cell-specific genetic variants and gene expression profiles, both of which have been shown to be related to the mechanisms of disease development, progression, and responses to treatment. Furthermore, stromal and immune cells within tumor microenvironment interact with cancer cells to play important roles in tumor responses to systematic therapy such as immunotherapy or cell therapy. However, most current high-throughput single cell sequencing methods detect only gene expression levels or epigenetics events such as chromatin conformation. The information on important genetic variants including mutation or fusion is not captured. To better understand the mechanisms of tumor responses to systematic therapy, it is essential to decipher the connection between genotype and gene expression patterns of both tumor cells and cells in the tumor microenvironment. We developed FocuSCOPE, a high-throughput multi-omics sequencing solution that can detect both genetic variants and transcriptome from same single cells. FocuSCOPE has been used to successfully perform single cell analysis of both gene expression profiles and point mutations, fusion genes, or intracellular viral sequences from thousands of cells simultaneously, delivering comprehensive insights of tumor and immune cells in tumor microenvironment at single cell resolution.

Disclosure Information Y. Arjmand Abbassi: None. N. Fang: None. W. Zhu: None. Y. Zhou: None. Y. Chen: None. U. Deutsch: None.

\section{P02.11 TREM1 AGONIST PY159 PROMOTES MYELOID CELL REPROGRAMMING AND UNLEASHES ANTI-TUMOR IMMUNITY}

V Juric*, E Mayes, M Binnewies, P Canaday, T Lee, S Dash, JL Pollack, J Rudolph, V Huang, X Du, N Jahchan, AJ Ramoth, S Mankikar, M Norng, C Santamaria, KP Baker, L Liang. Pionyr Immunotherapeutics Inc., South San Francisco, CA, USA
Background Tumor-associated myeloid cells can impede productive anti-tumor immunity. One strategy for targeting immunosuppression is myeloid reprogramming, which drives immunosuppressive myeloid cells to acquire an immunostimulatory phenotype. Triggering receptor expressed on myeloid cells-1 (TREM1) is an immunoglobulin superfamily cell surface receptor expressed on neutrophils and subsets of monocytes and tissue macrophages. TREM1 associates with DAP12 adaptor and induces proinflammatory signaling, amplifies innate immune responses, and is implicated in the development of acute and chronic inflammatory diseases. TREM1 is also enriched in tumors, specifically on tumorassociated myeloid cells. To investigate the potential of TREM1 modulation as an anti-cancer therapeutic strategy, we developed PY159, an afucosylated humanized antiTREM1 monoclonal antibody, and characterized it in the pre-clinical assays described below.

Materials and Methods An Fc $\gamma$ R binding ELISA and a Jurkat TREM1/DAP12 NFAT-luciferase reporter cell line were used to assess PY159 binding to human FcyRs and TREM1 signaling, respectively. PY159 responses in human whole blood in vitro were evaluated by flow cytometry, transcriptional analysis of sorted leukocyte subsets, and measurement of secreted cytokines/chemokines by MSD. A Transwell system was used to evaluate PY159 effects on neutrophil chemotaxis. TREM1 expression in human tumors was validated by scRNAseq, immunohistochemistry, and flow cytometry. Antitumor efficacy of a surrogate anti-mouse TREM1 antibody, PY159m, was evaluated using syngeneic mouse tumor models, either as a single agent or in combination with anti-PD1.

Results PY159 afucosylation increased its binding affinity for Fc $\gamma \mathrm{R}$ and its ability to activate TREM1/DAP12 signaling. In human blood assays, PY159 treatment did not induce depletion of TREM1-expressing cells. Rather, it upregulated monocyte activation markers, promoted neutrophil chemotaxis, and induced proinflammatory cytokines and chemokines, which was dependent on PY159 afucosylation. In human tumors, TREM1 was detected on tumor-associated neutrophils, tumor-associated macrophages, and monocytic myeloid-derived suppressive cells. PY159 induced proinflammatory cytokines and chemokines in dissociated human tumors in vitro, demonstrating that PY159 can reprogram tumor-associated myeloid cells. A surrogate anti-mouse TREM1 antibody, PY159m, exhibited anti-tumor efficacy in several syngeneic mouse tumor models, both as single-agent and in combination with anti-PD-1.

Conclusions These results show that PY159 is a TREM1 agonist that reprograms myeloid cells and unleashes anti-tumor immunity. PY159 safety and efficacy are currently being evaluated in first-in-human clinical trial (NCT04682431) involving patients resistant and refractory to standard of care therapies.

Disclosure Information V. Juric: A. Employment (full or parttime); Significant; Pionyr Immunotherapeutics Inc. E. Mayes: A. Employment (full or part-time); Significant; Pionyr Immunotherapeutics Inc. M. Binnewies: A. Employment (full or part-time); Significant; Pionyr Immunotherapeutics Inc. P. Canaday: A. Employment (full or part-time); Significant; Pionyr Immunotherapeutics Inc. T. Lee: A. Employment (full or part-time); Significant; Pionyr Immunotherapeutics Inc. S. Dash: A. Employment (full or part-time); Significant; Pionyr Immunotherapeutics Inc. J.L. Pollack: A. Employment (full or part-time); Significant; 
Pionyr Immunotherapeutics Inc. J. Rudolph: A. Employment (full or part-time); Significant; Pionyr Immunotherapeutics Inc. V. Huang: A. Employment (full or part-time); Significant; Pionyr Immunotherapeutics Inc. X. Du: A. Employment (full or parttime); Significant; Pionyr Immunotherapeutics Inc. N. Jahchan: A. Employment (full or part-time); Significant; Pionyr Immunotherapeutics Inc. A.J. Ramoth: A. Employment (full or parttime); Significant; Pionyr Immunotherapeutics Inc. S. Mankikar: A. Employment (full or part-time); Significant; Pionyr Immunotherapeutics Inc. M. Norng: A. Employment (full or part-time); Significant; Pionyr Immunotherapeutics Inc. C. Santamaria: A. Employment (full or part-time); Significant; Pionyr Immunotherapeutics Inc. K.P. Baker: A. Employment (full or part-time); Significant; Pionyr Immunotherapeutics Inc. L. Liang: A. Employment (full or part-time); Significant; Pionyr Immunotherapeutics Inc.

\section{P03 Vaccine Therapy}

\section{P03.01 HIGH IMMUNOGENIC VLP-BASED VACCINES ELICIT NEW T CELL SPECIFICITIES AGAINST MELANOMA NEOANTIGENS IN MICE}

${ }^{1} \mathrm{C}$ Aguilar-Gurrieri*, ${ }^{1} \mathrm{~A}$ Barajas, ${ }^{1} \mathrm{~A}$ Pons-Grifols, ${ }^{1} \mathrm{I}$ Varela, ${ }^{2} \mathrm{P}$ Amengual-Rigo, ${ }^{2} \mathrm{R}$ Farriol, ${ }^{2} \mathrm{M}$ Vazquez, ${ }^{2} \mathrm{R}$ Lepore, ${ }^{1} \mathrm{C}$ Anjos-Souza, ${ }^{1} \mathrm{~J}$ Blanco-Heredia, ${ }^{1} \mathrm{~L}$ de Mattos-Arruda, ${ }^{2,3} \mathrm{~V}$ Guallar, ${ }^{2} \mathrm{~A}$ Valencia, ${ }^{1,4} \mathrm{~B}$ Clotet, ${ }^{1} \mathrm{~J}$ Carrillo, ${ }^{1,4} \mathrm{~J}$ Blanco. ${ }^{1} / \mathrm{rs}$ iCaixa AIDS Research Institute, Badalona, Spain; ${ }^{2}$ Barcelona Supercomputing Centre (BSC), Barcelona, Spain; ${ }^{3}$ Catalan Institution for Research and Advanced Studies (ICREA), Barcelona, Spain; ${ }^{4}$ University of Vic-Central University of Catalonia (UVIC-UCC, Vic, Spain

\subsection{6/jitc-2021-ITOC8.24}

Background Neoantigens' (neoAg) identification, which determines T-cell responses against tumors, has fostered the development of personalized vaccines with promising results. While the ranking of the most immunogenic neoAg can be addressed using predictive techniques, their formulation as vaccines needs to be improved. To maximize their therapeutic potential, optimal neoAg-based vaccines should be manufactured in a superb delivery platform that enhances robust new immune responses, able to bypass thymic tolerance and the humoral immunosuppressive microenvironment. These novel $\mathrm{T}$ cell responses generated at the periphery will not be exhausted, opposite to TILs. We aim to develop a highly immunogenic vaccine platform, based on engineered HIV-derived Virus-Like Particles (VLP) expressing approximately 2500 copies of each selected neoAg. We tested different neoAgs loaded VLPs (neoVLP) in a melanoma mouse model to evaluate their capability to generate new immunogenic specificities.

Material and Methods Specific non-synonymous mutations from B16F10 cells were identified, selected and used to generate a list of prioritized peptides. NeoAgs were classified as: Tier1, acquiring a mutation that creates an anchor residue to the MHC-I, not present in the WT peptide; Tier2, acquiring a mutation in a position that largely impacts contact with the TCR respect to WT; and Tier3, acquiring a mutation in the TCR contact region but inducing a less drastic change than in Tier2. Frame shift (FS) mutations, expected to be highly immunogenic, were also included.Thirteen to fifteen selected neoAgs from each group were loaded on highly immunogenic neoVLPs. Their immunogenicity was evaluated in $\mathrm{C} 57 \mathrm{bl} / 6$ mice by immunization with a neoVLP-coding plasmid DNA (prime) and purified neoVLPs as soluble particles (boost). Splenocytes were used to evaluate neoAg-specific $\mathrm{T}$ cell responses.
Results We have successfully generated and purified neoVLPs, exposing neoAgs from all groups by transient transfection of Expi293 cells. Protein integrity and VLP morphology were confirmed by western blot and cryo-EM. When used for immunization assays, neoVLPs, containing neoAgs from Tier2, Tier3 and FS groups, were capable of generating humoral responses against viral proteins and $\mathrm{T}$ cell responses against neoAgs present in the neoVLP. B16F10 inoculated animals, but not vaccinated, did not develop detectable $\mathrm{T}$ cell responses against neoAgs present in any tested neoVLP, suggesting that the vaccination with neoVLPs promoted new specificities against selected neoAgs that might contribute to tumor control and eradication.

Conclusion Our data show that the neoVLPs promote the generation of new antitumor-specific immune responses against selected neoepitopes, suggesting that neoVLPs vaccination could be an alternative to current therapeutic vaccine approaches and a promising candidate for future personalized immunotherapy.

Disclosure Information C. Aguilar-Gurrieri: None. A. Barajas: None. A. Pons-Grifols: None. I. Varela: None. P. AmengualRigo: None. R. Farriol: None. M. Vazquez: None. R. Lepore: None. C. Anjos-Souza: None. J. Blanco-Heredia: None. L. de Mattos-Arruda: None. V. Guallar: None. A. Valencia: None. B. Clotet: F. Consultant/Advisory Board; Significant; Albajuna Therapeutics. J. Carrillo: Other; Significant; Albajuna Therapeutics. J. Blanco: F. Consultant/Advisory Board; Significant; Albajuna Therapeutics.

\section{P03.02 PROTEIN-BASED CANCER VACCINE COMBINED WITH AN ONCOLYTIC VACCINE PROMOTES POTENT ANTITUMOR IMMUNITY}

${ }^{1,2} \mathrm{E}_{\text {Belnoue }}{ }^{3,4}{ }^{3,4}$ Das, ${ }^{1,2} \mathrm{M}$ Rossi, ${ }^{3,4} \mathrm{~T}$ Hofer, ${ }^{3,4} \mathrm{~S}$ Danklmaier, ${ }^{5,2} \mathrm{~T}$ Nolden, ${ }^{3,4} \mathrm{~L}$ Schreiber, ${ }^{3,4} \mathrm{~K}$ Angerer, ${ }^{4} \mathrm{~J} \mathrm{Kimpel},{ }^{6} \mathrm{~S}$ Hoegler, ${ }^{6,7} \mathrm{~L}$ Kenner, ${ }^{3} \mathrm{D}$ von Laer, ${ }^{5,2} \mathrm{~K}$ Elbers, ${ }^{3,4} \mathrm{G}$ Wollmann, ${ }^{1,2} \mathrm{M}$ Derouazi. 'AMAL Therapeutics, Geneva, Switzerland; ${ }^{2}$ Boehringer Ingelheim International GmbH, Ingelheim, Germany; ${ }^{3}$ Christian Doppler Laboratory for Viral Immunotherapy of Cancer, Medical University of Innsbruck, Innsbruck, Austria; ${ }^{4}$ Institute of Virology, Medical University of Innsbruck, Innsbruck, Austria; ${ }^{5}$ Viratherapeutics GmbH, Innsbruck, Austria; ${ }^{6}$ Unit of Laboratory Animal Pathology, Institute of Pathology, University of Veterinary Medicine Vienna, Vienna, Austria; 'Department of Experimental Pathology, Medical University of Vienna, Vienna, Austria

\subsection{6/jitc-2021-ITOC8.25}

Background KISIMA ${ }^{\mathrm{TM}}$ platform allows the development of protein-based cancer vaccines able to induce a potent, tumorspecific CD8 and CD4 T cells response. While the cell penetrating peptide and peptide agonist for Toll like receptor (TLR)-2 and TLR-4 confer, respectively, the cell delivery and self-adjuvanticity properties, the multiantigenic domain allows the targeting of different cancer antigens, resulting in antitumoral efficacy in different murine models. Oncolytic viruses exert their therapeutic effects by a prolonged oncolytic action and the associated intratumoral inflammation as well as general immune activation. Arming oncolytic virus with tumor associated antigens can additionally enhance the tumor-specific $\mathrm{T}$ cell portion and therefore positively affect the balance of antitumor versus antiviral immune responses. The protein vaccine $\mathrm{KISIMA}^{\mathrm{TM}}$ and the recombinant oncolytic virus VSV-GP-TAA (vesicular stomatitis virus pseudotyped with LCMV GP expressing tumor-associated antigens) are both promising vaccine candidates that offer a new 